\title{
KURZMITTEILUNGEN
}

Z. klin. Chem. u. klin. Biochem.

7. Jg., S. 390 , Juli 1969

\section{Automatische enzymatische Galaktosebestimmung im Vollblut}

\section{Automatic enzymic determination of galactose in whole blood}

\section{Von J. D. Kruse-Jarres und V. KitngmülLer}

Klin. Chem. Institut, Klinikum Mannbeim der Universität Heidelberg (Direktor: Prof. Dr. Dr. V. Klingmüller)

(Eingegangen am 11. April 1969)

Kürzlich wurde in Arbeiten von TEngström und Mitarbeitern (1), HJELM und Mitarbeitern (2), RoMmel und Mitarbeitern (3) und MEHNERT und Mitarbeitern (4) auf die Bedeutung der Galaktosebelastungsprobe für die Differenzierung hepatogener Erkrankungen hingewiesen. $\mathrm{Da}$ sich durch eine neue Testpackung (TCGA 15291 TGAN Boehringer Mannheim $\mathrm{GmbH}$ ) eine spezifische und unkomplizierte Bestimmung der Galaktose anbietet, muß der sehr wesentlichen Abklärung von Lebererkrankungen durch Galaktosebelastungsproben eine zunehmende Bedeutung beigemessen werden.

\section{Methodik}

Wir stellen eine Methode vor, durch welche eine autoanalytische Bestimmung der Galaktose als UV-Test möglich ist. Die Bestimmung beruht auf der Reaktion:

Galaktose $+\mathrm{NAD}^{+} \frac{\text { Galaktose- }}{\text { Dehydrogenase }}$ Galaktonolacton + NADH $+\mathrm{H}^{+}$

Jeweils $3,4 \mathrm{ml}$ heparinisiertes Vollblut werden durch eine Rotationspumpe von einem Probennehmer (beide Fa. Technicon) im Rhythmus $40-2 / 1$ angesaugt. Jeder 2. Probenbecher ist mit

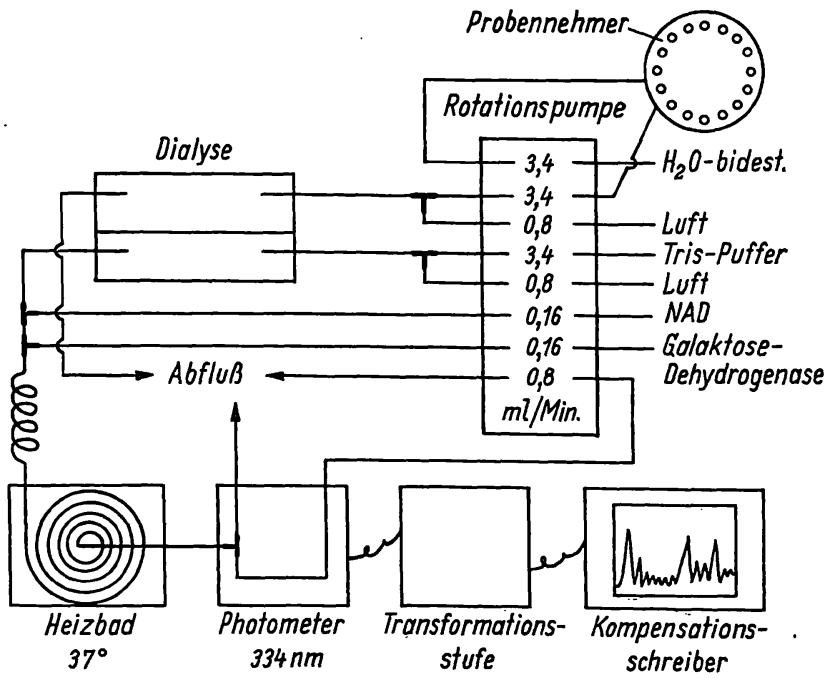

Abb. 1. Fli 2 Bdiagramm

dest. Wasser gefüllt, so daß sich eine Probenanalysenz̧ahl von 20/Std. ergibt. Das heparinisierte, ansonsten unverdünnte Vollblut wird in der anschließenden Dialyse ( $F$ a. Technicon) gegen einen $0,1 \mathrm{M}$ Tris-Puffer, $\mathrm{pH} 8,6$ dialysiert. Dem Dialysat wird anschließend zunächst NAD ( $4 \mathrm{~g} / \mathrm{l} 86 \% \beta$-NAD; Zufuhr $0,16 \mathrm{~m} / /$ Min.) und gleich darauf $\beta$-Galaktose-Dehydrogenase (EC 1.1.1.48) (etwa $5 \mathrm{U} / \mathrm{mg} ; 32,5 \mathrm{mg} /$; Zufuhr 0,16 ml/Min.) zugeführt. Dem nachfolgenden Heizbad ist eine Mischschlange vorgebaut. Im Heizbad ( $\mathrm{Fa}$. Technicon) wird das Reaktionsgemisch während einer Durchflußlänge von $12 \mathrm{~m}$ auf $37^{\circ}$ erwärmt. Es folgt die Messung der der Galaktosekonzentration äquivalenten NADHMenge in einer $10 \mathrm{~mm}$-Durchflußküvette durch das Photometer (Eppendorf) bei $334 \mathrm{~nm}$. Die Extinktion wird über eine Transformatorstufe $(E=0-0,25)$ vom Kompensationsschreiber (beide Eppendorf) aufgezeichnet (siehe Fließdiagramm).

Zur Eichung werden wäßr. Galaktoselösungen von 0,05, 0,10, $0,25,0,50,0,75,1,0,1,5$ und 2,0 g/l verwandt. Der Konzentrationsbereich bis $2,0 \mathrm{~g} / \mathrm{l}$ erweist sich bei oralen $(40 \mathrm{~g})$ sowie intravenösen $(0,33 \mathrm{~g} / \mathrm{kg} \quad \mathrm{KG})$ Galaktosebelastungen als ausreichend. Der Normalbereich bis $0,045 \mathrm{~g} / \mathrm{l}$ im menschlichen Blut kann mit dieser Methode gut erfaßt werden. Konzentrationen von $0,005 \mathrm{~g} / l$ sind noch meßbar.

\section{Ergebnișse}

Zur Bestimmung der Präzision werden Standardabweichung (s) und Variationskoeffizient (V) von 8 wäßr. Galaktoselösungen sowie 2 Blutproben jeweils 10 Bestimmungen in der Serie durchgeführt (s. Tab. 1). Dabei errechnet sich mit zunehmender Konzen-

Tab. 1

Standardabweichung und Variationskoeffizient

\begin{tabular}{ccc}
\hline $\begin{array}{c}\text { Galaktose } \\
\mathbf{g} / \mathrm{l}\end{array}$ & $\begin{array}{c}\text { Streuung der wieder- } \\
\text { gefundenen Galaktose } \\
\mathbf{g} / \mathrm{l}\end{array}$ & $\begin{array}{c}\mathrm{V} \\
\%\end{array}$ \\
\hline wäßrige Lösungen: & & \\
0,05 & $\pm 0,002$ & 4,0 \\
0,10 & $\pm 0,003$ & 3,2 \\
0,25 & $\pm 0,007$ & 2,8 \\
0,50 & $\pm 0,009$ & 1,8 \\
0,75 & $\pm 0,010$ & 1,3 \\
1,00 & $\pm 0,013$ & 1,3 \\
1,50 & $\pm 0,017$ & 1,1 \\
2,00 & $\pm 0,021$ & \\
Blutproben: & & 4,3 \\
0,046 & $\pm 0,002$ & 5,2 \\
0,038 & $\pm 0,002$ & \\
\hline
\end{tabular}

tration ein abnehmender Variationskoeffizient. Der relativ hohe Variationskoeffizient in Bereichen bis $0,1 \mathrm{~g} / l$ Galaktose resultiert aus der Wahl des sehr niedrigen Extinktionsbereiches $(0-0,25)$. Dieser ist mit der Übetlegung gewählt, die Kosten für das relativ teure Enzym und Coenzym durch entsprechende Verdünnungen ohne wesentliche Einbuße der Analysengenauigkeit so niedrig wie möglich zu halten und den Test somit als praktikabel in der Klinikroutine zu gestalten. Galaktose-Dehydrogenase und NAD müssen - um eine kọntinuierliche Zufuhr zu gewährleisten stark verdünnt werden und die Pumpenschläuche entsprechend groß gewählt werden, wenn davon ausgegangen wird, daß $3,4 \mathrm{ml}$ Vollblut/Min. zur Bestimmung verwandt werden. Nur so ist es möglich, mit dem Galaktose-Dehydrogenase-Anteil einer Testpackung $(0,65 \mathrm{ml}=3,25 \mathrm{mg})$ etwa $100-120$ Bestimmungen durchzuführen.

Uber die Möglichkeit kontinuierlicher Analysen in. Form von Galaktogrammen soll an anderer Stelle berichtet werden.

Der Fa. Boehringer Mannheim GmbH gilt unser Dank für die Galaktose-Dehydrogenase und das NAD. Der Fa. Technicon und der Deutschen Forschungsgemeinschaft sei Dank für apparative Unterstützung. Fräulein B. PApNer und Herrn R. BALKenHol danken wir für ihre technische Assistenz.

\section{Literatur}

1. Tengström, B., M. HJelm, C. H. de Verdier und I. Werner, Amer. J. Dig. Dis. 12, 853 (1967). - 2. HJELM, M. Clin. Chim. Acta, Amsterdam 15, 87 (1967). - 3. Rommel, K., E. Bernt, F. Schmitz und K. Grimmel, Klin. Wschr. 46, 936 (1968). - 4. Mehnert, H., M. Haslbeck und H. Förster, Dtsch. med. Wschr. 93, 1899 (1968).

Dr. J. D. Kruse-Jarres 68 Mannheim, Postfach 23 
Z. klin. Chem. u. klin. Biochem.

7. Jg., S. 391-392, Juli 1969

\section{Eine Einrichtung zur Darstellung und Photodokumentation} von Agar-Gel-Enzym-Elektropherogrammen

\section{Equipment for the visualisation and photodocumentation of agar gel electropherograms}

Von J. KaMarÝT

Aus dem Forscbungsinstitut für Pädiatrie,

(Direktor: Prof. MUDr. Z. Brunecky, C. Sc.) Brno (CSSR)

(Eingegangen am 9. Mai 1969)

Die Möglichkeit der densitometrischen Registrierung von Isoenzymaktivitäten, die mit dem Prinzip.des optischen Tests nach Warburg dargestellt wurden, mit Hilfe des Transparenz-Photometers bei $366 \mathrm{~nm}$, wird sehr häufig benutzt wegen ihrer Genauigkeit und Empfindlichkeit.

Während die Lichtabsorption von $\mathrm{NADH}_{2}$ im UV-Bereich, deren Zunahme oder Abnahme als Maß der Enzymaktivität dient, mit bloßem Auge unsichtbar ist, ist es möglich, für das Sichtbarmachen der Isoenzym-Fraktionen die zweite Eigenschaft, und zwar die Fluoreszenz des $\mathrm{NADH}_{2}$ auszunützen.

Die Agar-Gel-Schicht, welche $\mathrm{NADH}_{2}$ enthält, emittiert Fluoreszenz nach Bestrahlung mit UV-Licht (z. B. der Wellenlänge $366 \mathrm{~nm}$ ). In der Zone, in der die Enzymreaktion abgelaufen ist, nimmt diese Fluoreszenz ab infolge Oxydation des $\mathrm{NADH}_{2}$ oder verschwindet ganz. Die Fluoreszenz wird mit einer Quecksilberdampf-Hochdrucklampe angeregt; die emittierte Fluoreszenzstrahlung ermöglicht die Sichtbarmachung. Ohne Abschirmung des UV Lichtes, welches die Augen schädigt, ist aber die Beobachtung sehr schwierig.

Für die visuelle Beobachtung des Reaktions-Verlaufes im Agar-Gel wurde deshalb ein einfaches Gerät konstruiert, welches nach Abfiltrieren der emittierten Fluoreszenzstrahlung zugleich als Gerät für die Dokumentation der Enzym-Elektropherogramme auf Photopapier dient.

Das Gerät besteht aus dem Gehäuse, in das Peine Quecksilberdampf-Hochdrucklampe (HQV $125 \mathrm{~W}, 1,5 \mathrm{~A}$ ), deren Glaskolben ein Filter für das Licht der Wellenlänge $366 \mathrm{~nm}$ darstellt, eingebaut wird. Diese Lampe wird über eine Drossel angeschlossen und unten mit einem Reflektor ausgerüstet. Die Seiten des Gehäuses werden mit Kunststoffgittern verssehen, um die durch den Quecksilberbrenner entwickelte Wärme mit Hilfe eines kJeinen Ventilators absaugen zu können. Der Ventilator ist über einen Transformator und eine Germanium-Diode als Gleichrichter angeschlossen. Die Schalter für Ventilator und Quecksilberlampe befinden sich an der rechten Wand des Gehäuses. Der elektrische Aufbau des Gerätes ist aus Schema (Abb. 1) ersichtlich.

Das Gehäuse wird mit einem abnehmbaren Deckel, mit einer rechteckigen Offnung in der Mitte, ausgerüstet. Diese Offnung befindet sich direkt über der Entladungsbahn der $\mathrm{Hg}$-Lampe und ist mit einer Verschlußklappe, welche mit dem Drehknopf auf der rechten Seite des Gerätes bewegt wird, versehen. Auf dem Deckel befindet sich eine schwarze Platte, mit einem Fenster derselben Größe, wie die Deckel-Offnung. In dieses Fenster sind vier übereinander liegende gleiche Matt-Glasscheiben eingesetzt, die zur Lichthomogenisierung dienen. Uber der Platte mit dem Mattglasscheibensystem befindet sich eine zweite Platte mit.der Falzöffnung zum Einsetzen der Objektträger $(76 \times 26 \mathrm{~mm})$ mit der feuchten elektrophoretischen Agar-Gel-Schicht, welche mit der Detektionsschicht, die das entsprechende Substrat und $\mathrm{NADH}_{2}$ bzw. NAD enthält, bedeckt ist. In diesem Sandwich läuft die Enzym-Reaktion ab.

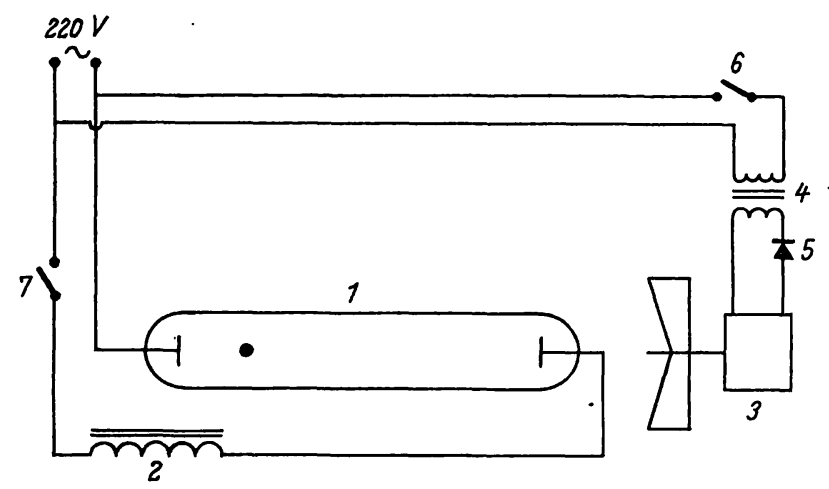

Abb. 1

Elektrischer Aufbau des beschriebenen Apparates

1. Quecksilberdampf-Hochdrucklampe HQV $125 \mathrm{~W}, 1,15$ A

2. Vorschaltgerät - Drosselspule

3. Ventilator $8 \mathrm{~V}$

4. Transformator $220 \mathrm{~V} / 8 \mathrm{~V}$

5. Gleichrichter - Germanium-Diod

. Ventilator-Schalter

7. Quecksilberdampf-Hochdrucklampen-Schalter

Nach Einschalten und Einbrennen der Hg-Lampe kann man in den Zonen, wo die Enzymreaktion abgelaufen ist oder noch abläuft, das Auslöschen der Fluoreszenz beobachten. Enzymbanden sind so als dunkle Fraktionen sichtbar.

Die photographische Dokumentation ist aber mit dieser Einrichtung nicht gut möglich. Für dieses Ziel dient der Zusatz, wclcher auf dem Schema (Abb. 2) mit der gestrichelten Linie gekenn-

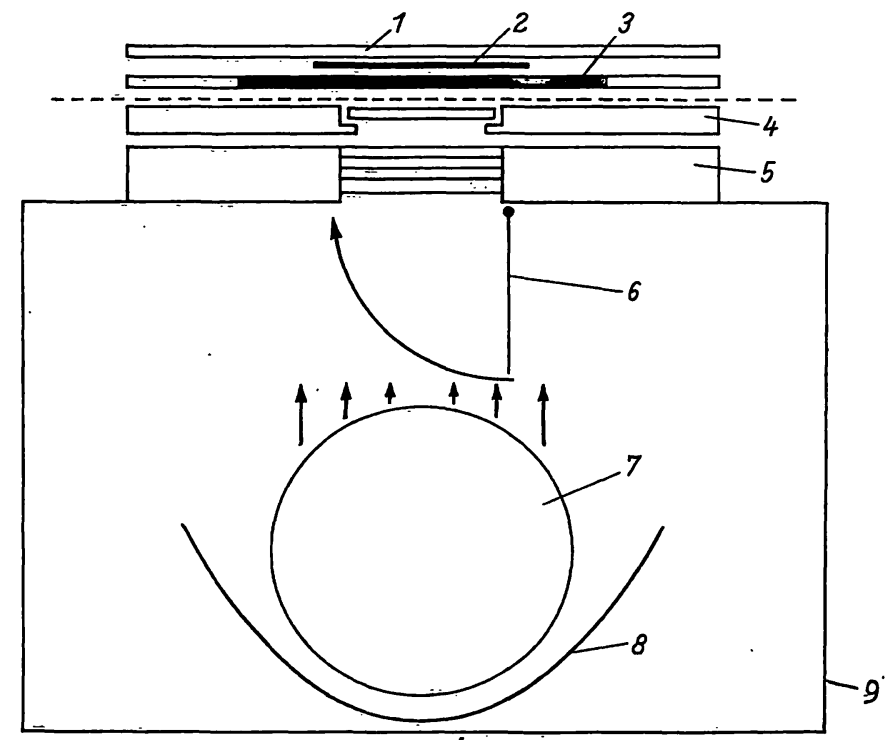

Abb. 2

Optischer Aurbau des beschriebenen Apparates

1. Andruckplatte für Photopapier

2. Photopapier

3. Platte mit Filter $366 \mathrm{~nm}$

4. Platte für Einsetzen des Enzym-Elektropherogramms

5. Platte mit dem Mattscheiben-System

6. Lichtverschlußklappe

7. Quecksilberdampf-Hochdrucklampe

8. Reflektor

9. Rehäruse

Die Teile 1, 2 und 3, welche mit der gestrichelten Linie gekennzeichnet sind, bilden den Ansatz für die Photodokumentation.

zeichnet wird. Die Platte mit dem Enzym-Elektropherogramm wird mit dem in eine dünne schwarze Kunststoffplatte eingebauten Glasfilter (Woodsches Glas - Schott, Jena - zur Isolierung der Wellenlänge $366 \mathrm{~nm}$ ) bedeckt. Auf das Filter legt man das Photopapier (Qualität Ultra-hart), welches durch eine zweite schwarze Kunstoffplatte fest angedrückt wird. Die Belichtung führt man durch kurzes Ein- und Ausschalten (ein Sekundenbruchteil) durch. Danach wird das Papier in einem kontrast arbeitenden Entwickler entwickelt. 

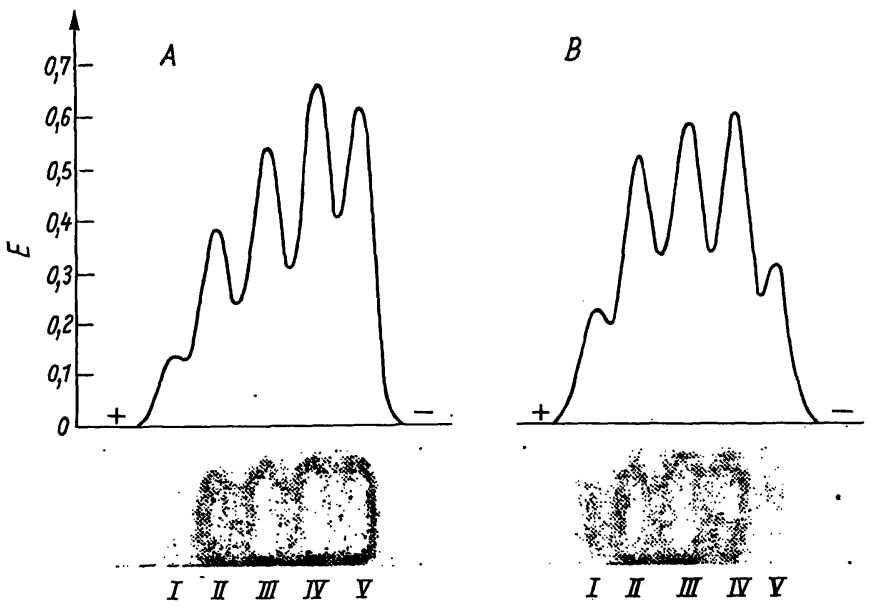

Als Beispiel wird die Dokumentation"der Lactatdehydrogenese und Aspartataminotranferase-Isoenzyme in Abbildung 3 gezeigt. Die Enzym-Elektropherogramme auf dem Photopapier kann man mit Hilfe geläufiger Densitometer mit Auflicht-Einrichtung qualitativ auswerten.

Das Gerät ermöglicht:

1. Visuelle Kontrolle des Reaktionsverlaufes (sofern der optische Test nach Warburg benutzt wird) vor der eigentlichen densitometrischen Registrierung der Aktivitäten bei der Wellenlänge $366 \mathrm{~nm}$.

2. Dokumentation der Enzym-Elektropherogramme auf Photopapier und die densitometrische Registrierung der Isoenzymaktivitäten im sichtbaren Wellenbereich (z. B. bei $550 \mathrm{~nm}$ ).

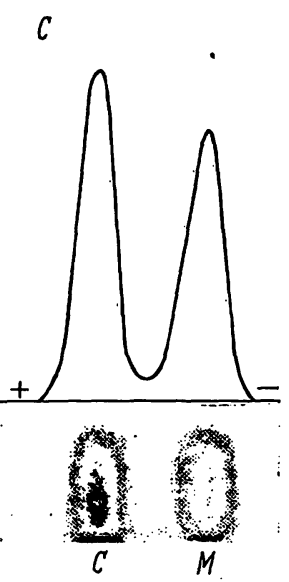

$\cdot 1$

Abb. 3

Dokumentation der LDH-(Lactatdehydrogenase) und GOT-(Glutamat-Oxalacetat-Transaminase)
Isoenzyme auf dem Photopapier Isoenzyme auf dem Photopapier
mit densitometrischer Registrie= rung $(550 \mathrm{~nm})$

A. LDH-Isoenzyme. Neutrophile Granulocyten. Akute myeloische

Leukämie-95,4\% Neutrophile. Lymphatische Leukämie $96,2 \%$ Lymphocyten.

C. Leberhomogenat. $C_{-}$cytoplasmatische Fraktion; $M-$ mitochondriale Fraktion der GOT.

Der Vorteil dieses Gerätes ist die volle Ausnutzung des optischen Tests nach Warburg für die Detektion der Isoenzymaktivitäten ohne Kopplung mit anderen Farbreaktionen. Diẹse IndikatorReaktionen sind kostspielig, nicht so empfindlich und schwache Isoenzymaktivitäten (wie z. B. Serum- $\mathrm{LDH}_{4}$ oder $-\mathrm{IDH}_{5}$ ) werden manchmal übersehen.

Die Gefahr der Augenschädigung des Laborarbeiters mit kurzwelligem Licht wird bei richtiger Handhebung des beschriebenen Geräts auf ein Minimum verringert.

RNDr. Jaromir Kamarýt, C. Sc. Forschungsinstitut für Pädiatrie Cerná Pole, Cernopolñi 9 Brno, Tschechoslowakei

\section{BUCHBESPRECHUNGEN}

Dekorporierung radioaktiver und stabiler Metallionen. Von A. Catsch. VIII, 57 Abb., 28 Tab., DM 32,-.

Verlag Karl Thiemig KG, München (1968).

Die Monographie von Alexander Carsch über die „Dekorporierung radioaktiver und stabiler Metallionen" befaßt sich auf 176 Seiten mit einer theoretisch sehr interessanten Arbeitsrichtung der experimentellen Medizin: Der Mobilisierung von radioaktiven Metallionen im Organismus durch Chelatbildner. Wenn auch die Fälle von Inkorporationen radioaktiver Metallionen selbst bei Reaktor-Arbeitern bisher extrem selten waren, ist es doch grundsätzlich bedeutsam, daß einiges theoretisches Rüstzeug für die Behandlung derartiger Fälle nunmehr vorliegt. Unter diesem Gesichtspunkt erklärt sich auch der Zusatz im Titel des Buches „Therapeutische Grundlagen“.

Dennoch liegt der Schwerpunkt der Monographie bei der Schilderung der experimentellen Grundlagen, so wie sie durch Tierversuche, hauptsächlich unter der speziellen Sicht des Autors, gewonnen wurden.

Während das vorliegende Buch im allgemeinen wohl nur für ausgesprochene Spezialisten in Frage kommt, ist die ausführliche Einführung in die Chemie der Chelatbildner für den anorganisch arbeitenden Biochemiker ein vielleicht interessanter Aspekt.

M. Wenzel, Berlin
FEBS 4th Meeting Proceedings Vol. 4: Cellular Compartmentalization and Control of Fatty Acid Metabolism. Hrsg. Gran. 116 S. 50 sh.

Academic Press Inc. (London) Ltd. (1968).

Dieses Buch enthält Vorträge, die am 5. Juli 1965 während der 4. Sitzung der "Federation of European Biochemical Societies" in Oslo gehalten wurden. Die Vorträge sind sehr gut überarbeitet gedruckt, enthalten eine Fülle von Ergebnissen, die durch Schemata und Tabellen anschaulich wiedergegeben werden und bieten darüber hinaus eine reichliche Literaturauswahl. Im einzelnen berichten F. LYNEN und Mitarbeiter über die Biosynthese der Fettsäuren, A. T. JAMES über die Biosynthese ungesättigter Fettsäuren in Photosynthesesystemen niederer und höherer Pflanzen, I. B. FrrTz über die Beeinflussung des Stoffwechsels durch die Wirkung des Carnitins auf die Oxydation langkettiger Fettsäuren, J. BREMER über die Faktoren, die die carnitinabhängige Oxydation von Fettsäuren beeinflussen und L. L. M. van DEENEN und Mitarbeiter über die strukturellen und metabolischen Aspekte bei Fettsäuren in Phosphoglyceriden. Die Qualität der Vorträge läßt entsprechend den angeführten Autoren nichts zu wänschen übrig. Das Buch vermittelt zweifellos eine Fülle neuer Ergebnisse und kann darüber hinaus sogar zum Nachschlagen empfohlen werden.

Die Wiedergabe von Gebrauchsnamen, Handelsnamen, Warenbezeichnungen und dgl. in dieser Zeitschrift berechtigt nicht zu der Annahme, daß solche Namen ohne weiteres von jedermann benützt werden dürften. Vielmehr handelt es sich häufig um gesetzlich geschützte Warenzeichen, auch wenn sie nicht eigens als solche gekennzeichnet sind.

(C) 1969 by Verlag Walter de Gruyter \& Co., vormals G. J. Göschen'sche Verlagshandlung J. Guttentag, Verlagsbuchhandlung · Georg Reimer Anzeigenverwaltung: Merkur-Werbung, Dr. K. Jeserich KG, 5213 Spich/Toisdorf, Merkur-Haus, Hauptstraße 23-27, Tel. (02 241) 77051, Anzeigenverwaltung: Merkur-Werbung, Dr. K. Jeserich KG, 5213 Spich/Toisdorf, Merkur-Haus, Hauptstraße 23-Her.
FS. 08/83386. Für den Anzeigenteil verantwortlich : Dr. Peter Bohrer, Spich/Troișdorf. 